\title{
Subject Retention in Prehospital Stroke Research Using a Telephone-Based Physician- Investigator Driven Enrollment Method
}

\author{
Bryant J. Rosell ${ }^{a} \quad K^{2}$ ristina Shkirkova ${ }^{a}$ Jeffrey L. Saver ${ }^{c}$ \\ David S. Liebeskind ${ }^{c, d}$ Sidney Starkman ${ }^{c}$ May Kim-Tenser ${ }^{a, b}$ \\ Marc Eckstein $^{a}$ Latisha Sharma ${ }^{c}$ Robin Conwite Scott Hamilton ${ }^{f}$ \\ Nerses Sanossian ${ }^{a, b}$ \\ a Keck School of Medicine, Department of Neurology, University of Southern California, \\ Los Angeles, CA, USA; ${ }^{b}$ Roxanna Todd Hodges Comprehensive Stroke Clinic, Department \\ of Neurology, University of Southern California, Los Angeles, CA, USA; 'Stroke Center, \\ Department of Neurology, University of California, Los Angeles, Los Angeles, CA, USA; \\ ${ }^{d}$ Neuroimaging Research Core, Department of Neurology, University of California, \\ Los Angeles, Los Angeles, CA, USA; ${ }^{\mathrm{e} N a t i o n a l ~ I n s t i t u t e ~ o f ~ N e u r o l o g i c a l ~ D i s o r d e r s ~ a n d ~}$ \\ Stroke, National Institutes of Health, Bethesda, MA, USA; ${ }^{f}$ Department of Neurology, \\ Stanford University, Palo Alto, CA, USA
}

Keywords

Stroke $\cdot$ Emergency medical services $\cdot$ Consent

\section{Abstract}

Background and Purpose: Subject retention into clinical trials is vital, and prehospital enrollment may be associated with higher rates of subject withdrawal than more traditional methods of enrollment. We describe rates of subject retention in a prehospital trial of acute stroke therapy. Methods: All subjects were enrolled into the NIH Field Administration of Stroke Therapy-Magnesium (FAST-MAG) phase 3 clinical trial. Paramedics screened eligible subjects and contacted the physician-investigator using a dedicated in-ambulance cellular phone. Physician-investigators obtained explicit informed consent from the subject or on-scene legally authorized representative (LAR) who reviewed and signed a consent form. Exception from informed consent (EFIC) was utilized in later stages of the study. Results: There were 1,700 subjects enrolled; 1,017 provided consent (60\%), 662 were enrolled via LAR (39\%), and 21 were enrolled via EFIC (1\%). Of the 1,700 patients, 1,413 (83\%) completed the 90-day visit, 265 (16\%) died prior to the 90 -day visit, and $22(1.3 \%)$ withdrew from the study before completion. There were no differences in rates of withdrawal by method of study enrolment, i.e., self-consent $(n=14), 1.4 \%$; LAR $(n=8), 1.2 \%$; EFIC $(n=0) 0 \%$. Conclusion: There was a high rate of retention when subjects were enrolled into prehospital stroke research using a phone-based method to obtain explicit consent. 
Informed consent is essential to ethical research in human subjects and it is regulated at many levels by the US Federal Government [1]. The method by which informed consent is obtained from patients can be critical to the optimization of subject retention in a study [2]. There are different means by which informed consent can be obtained from potential subjects. Consent can be granted by the patient, providing they are not significantly cognitively impaired. In circumstances where consent cannot be explicitly obtained from a competent subject, enrollment can proceed after obtaining consent from a legally authorized representative (LAR). If a LAR is not present and the subject is not competent to provide consent, the subject can be enrolled via exception from informed consent (EFIC) in emergency situations when approved by a local institutional review board. EFIC approval requires a period of public disclosure. In all cases of enrollment under EFIC, there must be an effort to obtain informed consent as soon as feasible.

The difficulty of providing clear, concise, and effective informed consent can be exacerbated when the consent must be given in the field during a medically emergent situation [3]. A unique challenge of conducting a prehospital study is the creation of a timely and efficient method of inclusion and exclusion criteria for potential study subjects [3]. When conducting a prehospital study, it is not possible for an investigative physician to screen all potential subjects in person. Therefore, a system needs to be devised in which EMS personnel are able to conduct the initial screening before contacting the physician-investigator, as well as a separate process for obtaining informed consent when deemed possible.

Recruitment and retention are dependent upon one another [2]. High rates of study drop out increase the costs of the study, but more importantly they have a negative effect on the validity of the data produced. Meeting the objectives of efficient recruitment and high retention in a prehospital study allows for the analysis of unprecedented interventions in medical emergencies. We aimed to describe the methods of enrollment and rates of subject retention in the NIH Field Administration of Stroke Therapy-Magnesium (FAST-MAG) clinical trial of prehospital-initiated neuroprotection.

\section{Methods}

The FAST-MAG clinical trial is a phase 3, NIH-NINDS-sponsored, randomized, placebocontrolled, clinical trial of field-initiated magnesium sulfate versus placebo in suspected hyperacute stroke patients within the first $2 \mathrm{~h}$ of the last known well time [4]. The detailed methods of this study have been previously published [5-7]. The clinical trial was active and enrolled in Los Angeles County from 2005 to 2012 and in Orange County from 2010 to 2012. There is a racially, ethnically, and socioeconomically diverse population of over 13 million people in Los Angeles and Orange Counties [8].

The great preponderance of patients in FAST-MAG are enrolled using explicit, written, and informed consent procedures. The consent process is a refined version of what was employed in the FAST-MAG pilot trial [9]. Explicit consent conversations are conducted by an off-site enrolling physician-investigator speaking by cellular phone with the consent provider, in parallel to paramedic delivery of on-site care. Consent conversations are conducted in a language in which the consent provider is fluent - either English or Spanish.

The consent provider is the patient if he or she is competent, or consent can be provided by the patient's on-scene LAR if the patient is not competent. When a patient or his or her representative indicates that they wish to learn more about the study, the paramedic hands them the appropriate English or Spanish language consent form approved by the patient's destination hospital. The physician completes study eligibility screening by confirming inclusion criteria and assessing for exclusion criteria, answers any questions the consent 
Fig. 1. Initial target population and final study population selection.

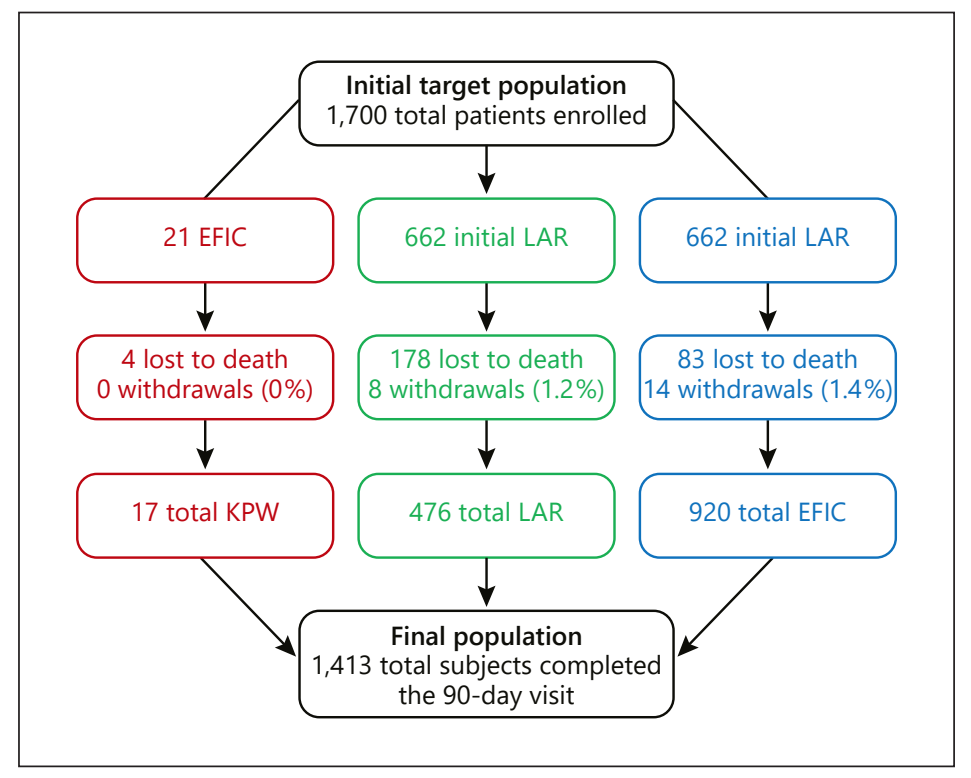

provider has regarding the study, and assesses the competence of the consent provider based on the individual's ability to restate key study procedures, risks, and what to do if they wish to discontinue participation. If the patient or their legally authorized representative agrees to participate in the trial, the consent provider signs the consent form in the field. Once the form is signed, the enrolling physician-investigator authorizes the paramedics to begin study agent infusion.

A small proportion of patients are enrolled in the FAST-MAG trial under federal regulations authorizing EFIC for research performed in emergency circumstances. This method of enrollment was added to the trial after launch to increase enrollment rates. Its use is confined to patients who are not competent to provide self-consent and who do not have on scene with them a LAR to provide proxy consent, but who do have on scene with them an informant who knows the patient well. In order to be able to enroll such patients, the FAST-MAG trial implemented the processes of community consultation and public disclosure required by EFIC regulations for hospitals in Los Angeles County. A community advisory panel was constituted with representatives from the Southern California Stroke Association, the American Stroke/ Heart Association, the American Association for Retired Persons, the National Association for the Advancement of Colored People, and the National Hispanic Coalition for the Elderly. Public meetings were held in catchment areas of the participating receiving hospitals (a total of 45 meetings attended by 2,069 individuals). Information about EFIC enrollment into the study was also disseminated to the public by print, radio, and television reports, community stroke education lectures, and the internet. Enrolling under EFIC was approved by 36 hospital sites and the Los Angeles EMS Agency.

\section{Results}

Of the 1,700 patients enrolled into the NIH FAST-MAG trial, consent was obtained directly from the subject in 1,017 (60\%) cases - from a LAR in 662 (39\%) and via EFIC in $21(1 \%)$ patients (Fig. 1). Out of these patients, 1,413 (83\%) completed the 90-day visit, 265 (16\%) died before completing the 90-day visit, and 22 (1.3\%) withdrew before completion. There 
was no significant difference in rates of withdrawal from the study by method of study enrollment (self-consent, $[n=14] 1.4 \%$; LAR, $[n=8] 1.2 \%$; and EFIC, $[n=0] 0 \%$ ). Of the 22 subjects who withdrew, 11 were lost to follow-up and 11 revoked consent directly or via their LAR. The most common cited reason for withdrawal of consent (63\%) was family objection to continued participation.

Language of consent was investigated as a potential reason for early study withdrawal. Consent was available in 2 languages, i.e., English and Spanish. Of the 1,700 patients enrolled, 1,505 (89\%) provided consent in English and 195 (11\%) provided consent in Spanish. Out of those enrolled in Spanish, 4 withdrew consent (2.1\%) versus 18 withdrawals $(1.2 \%)$ among those enrolled in English. There were no significant differences in rates of withdrawal by language of consent.

\section{Discussion}

We found that enrolling patients in a prehospital study via a phone-based method of obtaining explicit consent resulted in a high rate of retention. Among the study participants, method of enrollment (self, LAR, and EFIC) and language of enrollment (English vs. Spanish) were not significant factors for subject retention. Of those who withdrew from the study, the most often cited reason for withdrawal was family opposition to continued participation.

We believe the high rate of retention found in the NIH FAST-MAG clinical trial was a result of many systems working together. The enrollment process was clear, expedited, and sensitive $[1,3,10]$. Emergency medicine personnel across Los Angeles County was coordinated and trained to effectively screen and differentiate potential subjects $[3,11]$. Only a small number of physicians who were very familiar with study procedures were required to be available at all times to confirm and enroll a subject, either in Spanish or in English [3, 11]. There were many methods of consent available to cover many different situations that could be faced in the prehospital environment [3].

Limitations of this study include but are not limited to the following. This study did not include other languages common to the County of Los Angeles (Chinese, Tagalog, and Korean). Though withdrawal rates were low, further inquiries could have been made to ascertain the reasons for withdrawal and whether anything could have been done to further improve retention. These results may not be generalizable to populations outside of Los Angeles County and the surrounding area.

We ultimately believe prehospital enrollment to bean effective form of subject recruitment that enables the study of medical interventions at a time period where they are most likely to be effective.

\section{Statement of Ethics}

The authors have no ethical conflicts to disclose.

\section{Disclosure Statement}

David S. Liebeskind serves as a consultant for Stryker and Medtronic. 


\section{Funding Sources}

This study was supported by NIH-NINDS Award U01 NS 44364.

\section{References}

1 Lentz J, Kennett M, Perlmutter J, Forrest A. Paving the way to a more effective informed consent process: Recommendations from the Clinical Trials Transformation Initiative. Contemp Clin Trials. 2016 Jul;49:65-9.

2 Thayabaranathan T, Cadilhac DA, Srikanth VK, Fitzgerald SM, Evans RG, Kim J, et al. Maximizing Patient Recruitment and Retention in a Secondary Stroke Prevention Clinical Trial: Lessons Learned from the STAND FIRM Study. J Stroke Cerebrovasc Dis. 2016 Jun;25(6):1371-80.

3 Cofield SS, Conwit R, Barsan W, Quinn J. Recruitment and retention of patients into emergency medicine clinical trials. Acad Emerg Med. 2010 0ct;17(10):1104-12.

4 Saver JL, Starkman S, Eckstein M, Stratton SJ, Pratt FD, Hamilton S, et al.; FAST-MAG Investigators and Coordinators. Prehospital use of magnesium sulfate as neuroprotection in acute stroke. N Engl J Med. 2015 Feb; 372(6):528-36.

5 Saver JL, Starkman S, Eckstein M, Stratton S, Pratt F, Hamilton S, et al.; FAST-MAG Investigators and Coordinators. Methodology of the Field Administration of Stroke Therapy - Magnesium (FAST-MAG) phase 3 trial: Part 2 - prehospital study methods. Int J Stroke. 2014 Feb;9(2):220-5.

6 Saver JL, Starkman S, Eckstein M, Stratton S, Pratt F, Hamilton S, et al.; FAST-MAG Investigators and Coordinators. Methodology of the Field Administration of Stroke Therapy - Magnesium (FAST-MAG) phase 3 trial: Part 1 - rationale and general methods. Int J Stroke. 2014 Feb;9(2):215-9.

7 Sanossian N, Starkman S, Liebeskind DS, Ali LK, Restrepo L, Hamilton S, et al.; FAST-MAG Trial Investigators. Simultaneous ring voice-over-Internet phone system enables rapid physician elicitation of explicit informed consent in prehospital stroke treatment trials. Cerebrovasc Dis. 2009;28(6):539-44.

8 Lofquist D, Lugiala T, O'Connell M, Feliz S. Households and Families: 2010. 2010 Census Briefs, US Census Bureau (http://wwwcensusgov/prod/cen2010/briefs/c2010br-14pdf) 2010.

9 Saver JL, Kidwell C, Eckstein M, Ovbiagele B, Starkman S; FAST-MAG Pilot Trial Investigators. Physician-investigator phone elicitation of consent in the field: a novel method to obtain explicit informed consent for prehospital clinical research. Prehosp Emerg Care. 2006 Apr-Jun;10(2):182-5.

10 Villamañán E, Ruano M, Fernández-de Uzquiano E, Lavilla P, González D, Freire M, et al. Informed consent in clinical research; Do patients understand what they have signed? Farm Hosp. 2016 May;40(3):209-18.

11 Carey TS, Kinsinger L, Keyserling T, Harris R. Research in the community: recruiting and retaining practices. J Community Health. 1996 Oct;21(5):315-27. 